ARTICLE

\title{
Perinatal management of spina bifida
}

\author{
L Padayachy, ${ }^{1} \mathrm{MB}$ ChB, MMed, FCNeurosurg (SA); D Ochieng', ${ }^{2} \mathrm{MB} \mathrm{ChB}$ \\ ${ }^{1}$ Division of Neurosurgery, University of Cape Town and Spina Bifida Clinic, Red Cross War Memorial Children's Hospital, Cape Town, South Africa \\ ${ }^{2}$ Division of Neurosurgery, University of Cape Town, South Africa
}

Corresponding author: L Padayachy(l.padayachy@uct.ac.za)

The management of patients with myelomeningocele is largely dependent on the timing of the diagnosis, i.e. ante- or postnatally. Antenatal diagnosis can be made using a combination of maternal serum alpha-fetoprotein measurement, fetal ultrasonography and, where necessary, amniocentesis.

S Afr Med J 2014;104(3):219. DOI:10.7196/SAMJ.8038

The management of patients with myelomeningocele (MMC) is largely dependent on the timing of the diagnosis, i.e. ante- or postnatally. As previously discussed in $C M E,^{[1]}$ antenatal diagnosis can be made using a combination of maternal serum alpha-fetoprotein (MSAFP) measurement, fetal ultrasonography and, where necessary, amniocentesis. An elevated MSAFP should be repeated, and if both values are elevated, fetal ultrasound, which has a sensitivity of $90 \%$, is recommended as the next step. ${ }^{[2]}$ In the modern era, with routine antenatal screening, the majority of cases should be detected before delivery.

Adequate counselling of parents is incumbent on the treating doctor. The complexity of the diagnosis and its sequelae can be quite overwhelming for the family. It is therefore very important to discuss thoroughly the nature of the abnormality, as well as the possible consequences. The decision by the family to either continue with the pregnancy or to opt for termination of pregnancy, usually involves a complex mix of personal and religious factors, but may be strongly influenced by the advice of the treating doctor. With appropriate medical care, the majority of patients with this condition can enjoy a good quality of life. The current accepted mortality in patients with MMC is about $25 \%$, with $15 \%$ dying in the first 5 years of life. ${ }^{[3]}$ When the decision to continue with the pregnancy has been made, the next step involves choosing the most appropriate manner of obstetric management.

\section{Obstetric management}

Once the diagnosis has been made antenatally, consideration should be given to the mode of delivery, i.e. vaginally or by caesarean section. Some clinicians recommend caesarean delivery before rupture of the amniotic membranes and the onset of labour in order to reduce injury and prevent infection of the MMC sac. However, long-term ambulation status in infants with MMC who underwent a trial of labour was no different than in those who underwent elective caesarean delivery. ${ }^{[4]}$ Although parents who elect to continue with the pregnancy may prefer a planned caesarean section to optimise postnatal management, the decision as to mode of delivery should be made on the basis of accepted obstetric criteria.

\section{Immediate postnatal care}

The main priorities are protecting the open lesion to prevent infection and detecting brainstem dysfunction related to the Chiari II malformation, with a view to referring the newborn for surgical closure within 24 hours. Immediate assessment and management should include:

- ABCs, APGAR score, adequate resuscitation, noting stridor or any other brainstem signs

- evaluating the lesion with regard to

- size

- level

- content

- checking for associated congenital abnormalities (vertebral defects, anal atresia, cardiac defects, tracheo-oesophageal fistula, renal anomalies, and limb abnormalities (VACTERL syndrome)).

The lesion should be covered with a warm saline-soaked dressing to protect the placode and prevent desiccation. Although all MMCs are open lesions, and the most effective antimicrobial treatment is early surgical closure, some authors believe that an MMC that leaks cerebrospinal fluid (CSF) should be considered 'ruptured' and has a higher risk of infection. Because of the latter, they recommend the administration of antibiotics, preferably with Gram-negative and anaerobic cover, e.g. ceftriaxone and gentamicin. As thermoregulation is a major challenge, the infant should be nursed prone in an incubator. The infant usually commences breastfeeding, but a nasogastric tube may be required if there is evidence of swallowing dysfunction or aspiration. As latex allergy is a long-term problem, all latex products must be avoided from the outset. Further investigations may include a swab for culture of the open lesion as well as blood for determining the international normalised ratio (INR), full blood count (FBC) and cumulative urinary excretion (CUE).

Once the neonate is stable, further neurological assessment must be done:

- measurement of the head circumference and assessment of the anterior fontanelle to check for hydrocephalus

- ascertaining the highest level of motor function by observing spontaneous movement of the lower limbs (Tables 1 and 2)

- assessment of anal tone by evaluating the muscle bulk of the buttocks and whether the anus is patulous

- looking for signs related to Chiari II malformation, e.g. apnoea, stridor and swallowing difficulties.

Referral for neurosurgical closure should take place within 24 hours, or at most 48 hours. If delayed, there is a much greater likelihood that the lesion will be colonised and infected. 
Table 1. Assessment of motor function

\begin{tabular}{ll}
\hline Spinal segment tested & Muscle function \\
\hline L2, 3 & Flexion at the hip joint \\
L3, 4 & Extension at the knee joint \\
L4, 5 & Dorsiflexion at the ankle joint \\
L5, S1 & Flexion at the knee joint, extension of the halux \\
S1, 2 & Plantar flexion at the ankle joint \\
S2, 3 & Flexion of the hallux and toes \\
S2, 3, 4 & Anal sphincter tone or wink
\end{tabular}

Table 2. Assessment of sensory function

\begin{tabular}{ll}
\hline Spinal segment tested & Dermatome \\
\hline L1 & Inguinal crease \\
L2 & Femoral triangle \\
L3 & Knee \\
L4 & Medial leg \\
L5 & Lateral leg and dorsum of foot
\end{tabular}

\section{Surgical management}

The current neurosurgical management of a child with MMC represents one component of a multidisciplinary approach, and can best be divided into three phases: (i) closure of the MMC; (ii) treatment of the associated hydrocephalus; and (iii) longterm surgical management.

\section{Closure of the myelomeningocele}

Initial management of the patient should follow the protocol described above. The closure should ideally be done within 24 hours of birth, but if it is delayed by more than 72 hours, the risk of the wound becoming colonised and infected increases substantially. Swabs of the wound should be taken and sent for microscopy and culture, and appropriate antibiotic therapy commenced where necessary. A history of prolonged rupture of the membranes antenatally may be another indication to commence antibiotic therapy. Three clean swabs, i.e. no organisms cultured, are required before surgical closure of the defect can be performed.

Careful neurological examination of the neonate must be done prior to surgery so that any improvement in or worsening of the neurological deficit can be documented. A swab of the lesion should be taken in theatre, prior to closure. The surgical procedure must be done under general anaesthesia with adequate monitoring and appropriate intravenous access. The main benefit of early closure is prevention of infection and preservation of neurological function.

The surgical procedure involves careful dissection of the neural placode, protecting any functional nerve roots. Where possible, the placode is then reconstituted, followed by a careful, layered, watertight closure of the dura and myofascial tissue. Careful repair of the malformation may reduce the risk of subsequent tethering. The skin surrounding the lesion can be quite friable and, depending on the complexity of the defect, related procedures can range from simple re-approximation through to complex reconstruction.

Postoperative management requires meticulous care of the wound and dressing, also to prevent wound breakdown. These neonates should be nursed prone, with the nappy open to prevent faecal soiling of the wound. Hydrocephalus occurs in $80-90 \%$ of cases, caused in part by closure of the MMC, but mainly by the associated Chiari II malformation. ${ }^{[5,6]}$ Daily head circumference measurements are mandatory to detect progressive ventricular enlargement, which can be supplemented by regular ultrasound examination, deferring the need for a computed tomography (CT) scan and the accompanying radiation. Occasionally, temporary CSF drainage may be required to prevent leakage from the MMC closure site. Apnoea monitors should be used for early detection of compromised respiratory function related to the Chiari II malformation.

A very important component of the management at this stage involves counselling of the family by a social worker, as the social and financial implications for the family need to be evaluated. It is also helpful to inform the family of the assistance available to them in terms of social grants, occupational therapy and special needs education.

The bladder function should be evaluated by early referral to a urologist. Although these infants are usually incontinent, acute urinary retention has been described, particularly after insertion of a ventriculoperitoneal shunt (VPS).

Intrauterine fetal surgery for closure of an MMC has demonstrated some benefit as suggested in the MOMS study. ${ }^{[7]}$ It appears to decrease the rate of shunt dependence at 1 year of age, reduces the risk of fetal or neonatal death, and improves the motor outcome. It does, however, also pose substantial risks to the mother and fetus. Appropriate selection criteria for and optimal timing of intrauterine surgery remain issues of contention.

\section{Treatment of hydrocephalus}

Hydrocephalus may be apparent at the initial assessment or may become more apparent days or weeks after closure of the MMC. When hydrocephalus is obvious at birth, a CSF diversion procedure, usually an external ventricular drain (EVD), can be inserted at the time of the MMC closure. Some reports suggest that contemporaneous insertion of a VPS with MMC closure is not associated with any increased risk, ${ }^{[8]}$ but this is not a widely accepted practice.

Our current management protocol favours delayed VPS insertion once the MMC wound has completely healed - usually around two weeks after closure. Monitoring of hydrocephalus during this period can be comfortably achieved with daily head circumference measurements and ultrasound assessment of ventricular size. Ventricular taps (maximum of three taps) and EVD insertion can be used as temporary measures to control the hydrocephalus. VPS insertion is necessary in the majority of MMC patients; however, the malfunction rate of these shunts is high, with a third requiring revision within the first year. Endoscopic third ventriculostomy (ETV) has also been described in this setting, but has not enjoyed much success. ${ }^{[9]}$ ETV combined with choroid plexus cautery appears quite promising in certain cases. ${ }^{[10]}$

\section{Long-term surgical management}

A multidisciplinary team comprising a neurosurgeon, an orthopaedic surgeon, a urologist, a physiotherapist, an occupational therapist and a nurse co-ordinator should follow up the child with an MMC. This follow-up will continue throughout childhood, and often into adulthood. The role of the neurosurgeon largely involves monitoring of the hydrocephalus or checking for shunt malfunction, symptoms related to the Chiari II malformation, and symptoms and signs of spinal cord tethering.

\section{Conclusion}

The perinatal management of children born with an MMC remains a challenging task, with early identification and timeous referral to specialist centres having a significant impact on survival. 


\section{References}

1. Wegoye E, Padayachy L. Recognising anomalies of the nervous system. CME 2013;31(3):107-109.

2. Boyd PA, Devigan C, Khoshnood B, et al. Screening policies in Europe for structural malformations and chromosome anomalies, and their impact on detection and termination rates for neural tube defects and Down's syndrome. Br J Obstet Gynaecol 2008;115(6):689-696. [http://dx.doi.org/10.1111/ j.1471-0528.2008.01700.x]

3. Bowman RM, McLone DG, Grant JA, et al. Spina bifida outcome: A 25 year prospective. Pediatr Neurosurg 1999;9:5-8

4. Lewis D, Tolosa JE, Kaufmann M, Goodman M, Farrell C, Bergvella V. Elective cesarean delivery and long-term motor function or ambulation status in infants with meningomyelocoele. Obstet Gynecol 2004;103(3):469-473. [http://dx.doi.org/10.1097/01.AOG.0000113624.94710.ce]

5. Lorber J. Results of treatment of myelomeningocoele: An analysis of 524 unselected cases, with special reference to possible selection for treatment. Dev Med Child Neurol 1971;13:279-303.
6. McLone DG, Dias L, Kaplan WE, et al. Concepts in the management of spina bifida. In: Humphreys RP, ed. Concepts in Pediatric Neurosurgery. Vol 5. Basel: S Karger, 1985:97-106.

7. Adzick NS, Thom EA, Spong CY, Brock JW, Burrows PK. A randomized trial of prenatal versus postnat repair of myelomeningocele. N Engl J Med 2011;364:993-1004. [http://dx.doi.org/10.1056/NEJMoal014379] 8. Parent $\mathrm{AD}$, McMillan T. Contemporaneous shunting with repair of myelomeningocoele. Pediat Neurosurg 1995;22:132-135. [http://dx.doi.org/10.1159/000120890]

Teo C, Jones R. Management of hydrocephalus by endoscopic third ventriculostomy in patients with myelomeningocoele. Pediatr Neurosurg 1996;25:57-63. [http://dx.doi.org/10.1159/000121098]

10. Warf BC, Campbell JW. Combined endoscopic third ventriculostomy and choroid plexus cauterization as primary treatment of hydrocephalus for infants with myelomeningocele: Long-term results of a prospective intent to treat study in 115 East African infants. J Neurosurg Pediatr 2008:2(5):310-316. [http://dx.doi.org/10.3171/PED.2008.2.11.310] 